\title{
Zur Kenntnis der Überwallungsharze
}

(IX. Abhandlung)

Von

Max Bamberger und Herbert v. Klimburg

Aus dem Laboratorium für anorganische Chemie an der k. k. Technischen Hochschule in Wien

(Vorgelegt in der Sitzung am 3. Mai 1917)

\section{Das Über wallungsharz der Zirbe.}

Es wurde bereits in einer Reihe von Mitteilungen über die Zusammensetzung der Überwallungsharze der Schwarzföhre, Fichte und Lärche berichtet. ${ }^{1}$ Gelegentlich einer Exkursion in das Aferertal (Palmtschoss), Südtirol, konnte eine kleine Quantität des Harzes der Zirbe gesammelt werden, welches die typischen Eigenschaften der Überwallungsharze aufwies.

Herr Prof. Dr. F. Krasser hatte die besondere Güte, die naturhistorische Charakterisierung des in Rede stehenden Rohstoffes im Nachfolge nden zusammenzufassen.

1 1. M. Bamberger, Monatshefte für Chemie, 12, 441, (1891).

2. $>>$ ebenda, 15, 505, (1894).

3. M. Bamberger und A. Landsiedl, ebenda, 18, 481, (1897).

4. > $>>>20,647,(1899)$.

5. \ > > $>20,755,(1899)$.

6. $>$ E. Vischner, $\gg$ 21, 564, (1900).

7 . > $>>>21,949,(1900)$.

H. Hermann, ebenda, 23, 1022, (1902).

8. M. Bamberger und H. Renezeder, ebenda, 24, 209, (1903). 


\section{Überwallungsharz der Zirbe.}

»Krümelige, bis tränenartige oder krustige Überzüge auf und zwischen den Borkeschuppen von bernsteingelber Farbe, deren rauhe Oberfläche schließlich meist weiß bestäubt erscheint.

\section{Mikroskopischer Befund:}

Im auffallenden Lichte. Häufungen kleiner milchweißer Körner und Kryställchen und größerer topasgelber Krystalle. Die weißen Kryställchen sitzen den gelben oft un deutlich geformten großen Krystallen auf und sind auch $z$ wischen letztere gelagert.

Im durchfallenden Lichte.

1. Der zähflüssige Balsam: In homogener Grundmasse sind Körnchen- und Nadelgruppen, sowie teils unregelmäßige, teils regelmäßige Täfelchen erkennbar. Die Täfelchen selbst sind von körniger oder körnig-nadeliger Struktur. Vereinzelt werden wetzsteinförmige Krystalle beobachtet. Als accessorische Elemente finden sich Pilzhyphen, Pilzsporen, auch Rindenalgen, Moosprotonemen und Koniferenpollen, sowie Staubpartikelchen. Im dunklen Gesichtsfelde des Polarisationsmikroskops erscheinen die Harzelemente teils in bläulicher, teils in gelblicher Färbung.

2. Die härteste bernsteingelbe Masse: Iṇ Luft präpariert erweist sie sich, im durchfallenden Lichte betrachtet, als ein Konglomerat aus großen Schollen verschiedener Gestalt, die von feinen Nädelchen und Krümelchen bedeckt sind. Schon bei Abstellung des Spiegels, also im dunklen Gesichtsfeld und bei auffallendem Lichte, tritt die gelbe Färbung der Schollen und die milchweiße ihrer Bedeckungsgebilde hervor.

Wird als Beobachtungsmedium für die mikroskopische Betrachtung des Überwallungsharzes der Zirbe Terpentinöl oder Zirbelkieferöl (Muster von Brüder Unterweger, Tirol 1894) in Anwendung gebracht, so treten die geschilderten, morphologischen Verhältnisse scharf hervor. Im polarisierten Lichte erscheinen die Bedeckungskryställchen sehr hell und in ver* schiedenen Polarisationsfarben, die großen Krystalle hingegen matt. 
Schwefelsäure bewirkt keinerlei Farbenreaktion der Harzkonstituenten. Das Harzpulver ändert an der Luft seine gelblichweiße Farbe nicht.

Im Harze vorkommende rote oder rotbraune Partikelchen sind stets als Fremdkörper erkennbar und spärlich."

\section{Aufarbeitung des Zirbenharzes.}

Zur Untersuchung wurden zirka $5 \mathrm{~kg}$ des oben beschriebenen Harzes verwendet.

Das Rohharz hatte einen angenehmen aromatischen Geruch, der an Vanilin und vielleicht auch an etwas freie Buttersäure erinnerte. Besonders im gepulverten Zustande trat letzterer Geruch stärker hervor.

Das Reinharz, das durch Lösen des Rohharzes in Alkohol und Eingießen dieser Lösung in mit Salzsäure angesäuertes Wasser erhalten wurde, ist beinahe weiß. Durch Einwirkung des Lichtes wird es bald rotgefärbt und ist nach zirka zwei Tagen dunkelrotbraun. Diese Farbenänderungen treten schneller in trockenem als im feuchten Zustande auf. Im Vakuumexsikkator ist es bereits nach. wenigen Stunden rotbraun.

Der Schmelzpunkt des Harzes liegt bei zirka $70^{\circ}$. Es löst sich leicht in Äthylalkohol, Methylalkohol, Aceton, Tetrachloräthan und teilweise in Äther, Benzol, Chloroform und Schwefelkohlenstoff.

Die Säure- und Jodzahl wurde nach der Methode von Schmidt und Erban ${ }^{1}$ ermittelt. An eine Bestimmung der Köttstorfer'schen Zahl konnte nicht gedacht werden, da die alkalische Lösung des Harzes so dunkel gefärbt war, daß ein Titrieren ausgeschlossen oder zumindest höchst ungenau gewesen wäre.

Säurezahl ..... 127

Jodzahl ....... 112 (und $78 \cdot 4$ )

Die Jodzahl zeigte starke Schwankungen. Bei den natürlichen bernsteingelben und auch etwas dunkler gefärbten

1 Monatshefte für Chemie, 7, 655, (1886). 
Harztropfen betrug sie 112, hingegen hatte das reine ausgekochte Harz die geringere Jodzahl von nur $78 \cdot 4$.

Der Gehalt an Methoxyl ist im Zirbenharz ein geringerer als in den anderen Überwallungsharzen.

In 1000 Teilen Überwallungsharz sind Teile Methoxyl:

1. Zirbe .......... 13

2. Fichte ......... $30-35$

3. Lärche .......... 45

4. Schwarzföhre ....... 50-55

Um zu ermitteln, ob das Harz beim Kochen mit Wasser an dieses Substanzen abgibt, wurde es samt der Borke mit diesem übergossen und längere Zeit gekocht, die Lösung vom geschmolzenen Harz und der Borke abfiltriert und mit Äther ausgeschüttelt. Beim Verdunsten des letzteren hinterblieben viele kleine, gut ausgebildete Krystalle, die sichelförmig gekrümmt und garbenartig verwachsen waren. Neben diesen Krystallen waren noch andere Formen vorhanden.

Zur Darstellung einer größeren Menge dieses Rückstandes wurde nun die ganze oben erwähnte Menge von $5 \mathrm{~kg}$ aufgearbeitet. Ein Trennen des Harzes von der Borke auf mechanischem Wege war ausgeschlossen, da das Harz über diese und zwischen dieser verteiit war. Daher wurde das Harz samt der Borke mit zirka $10 l(95 \%)$ Alkohol übergossen, einige Tage stehen gelassen und hierauf die Lösung von der Rinde abfiltriert. Aus dem Filtrat konnte der Alkohol in mehreren Partien durch Einleiten von Wasserdampf entfernt werden, durch welche Operation das Harz am Boden des Kolbens als lichte, braunrote, geschmolzene Masse abgeschieden wurde. Mit dem Einleiten von Wasserdampf wurde aufgehört, als das aus dem Kolben übergehende Destillat nicht mehr nach Alkohol roch, sondern nur mehr ein Geruch nach Terpentin oder Latschenöl wahrzunehmen war. Die über dem geschmolzenen Harz befindliche wässerige Lösung wurde abfiltriert und für sich untersucht. Diese sei mit $A$, das geschmolzene Harz mit $B$ bezeichnet. 


\section{Aufarbeitung von $A$.}

Aus der wässerigen Lösung schied sich nach kurzem Stehen ein harzartiger Körper ab. Eine getrennte Untersuchung dieser Abscheidung und der wässerigen Lösung hatte sich schon bei früheren Arbeiten als unnötig erwiesen, daher wurden beide zugleich mit Äther wiederholt ausgeschüttelt und letzterer abdestilliert. Es hinterblieb im Kolbẹn eine dicke, braune Masse, die selbst nach längerer Zeit nicht die geringste Neigung zu krystallisieren zeigte. Jeder Versuch, diese Masse aus irgend einem passenden Lösungsmittel zum Krystallisieren zu bringen, war vergebens. Eisenchlorid erzeugte in der wässerigen Lösung der so gewonnenen Substanz eine dunkelgrüne Färbung, Bleizucker gab in der alkoholischen Lösung dieser syrupartigen Masse einen reichlichen, gelben Niederschlag. Auf Grund dieser Fällung wurde nun eine ähnliche Methode, wie sie auch Hlasiwetz und Barth ${ }^{1}$ bei ihren Arbeiten zur Abscheidung der im Harz präformiert enthaltenen Substanzen benützten, verwendet.

Der früher nach Abdestillieren des Äthers erhaltene Destillationsrückstand gab in Alkohol aufgenommen und mit einer hinreichenden Menge von Bleizucker in alkoholischer Lösung versetzt einen reichlichen, voluminösen, geiben Niederschlag, der abgesaugt und mit Alkohol und Äther nachgewaschen wurde. Das zurückbleibende, gut mit Wasser verriebene Bleisalz wurde mit Schwefelwasserstoff zersetzt und nach dem Abfiltrieren des Bleisulfids ergab die wässerige Lösung beim Einengen reichliche krystallinische Abscheidung. Ein reineres Produkt in größeren Krystallen konnte erhalten werden, wenn die wässerige Lösung nicht direkt zum Krystallisieren gebracht, sondern wiederholt mit Äther ausgeschüttelt wurde. Nach dem Verdunsten des Äthers hinterblieben schöne große Krystalldrüsen, die aus monoklinen, gelben, prismatischen Nadeln zusammengesetzt waren. Die wässerige Lösung derselben gab mit Eisenchlorid eine grüne Färbung, die auf Zusatz von Soda dunkelrot wurde. Die wässerige Lösung reduziert Silbernitrat. Bleizucker gibt mit

1 Liebig's Annalen, 134, 265, (1865). 
derselben einen zitronengelben Niederschlag. Alle diese Eigenschaften erinnern sehr an Kaffeesäure, die schon in einer früheren ${ }^{1}$ Untersuchung im Überwallungsharz der Schwarzföhre aufgefunden wurde. Um nun diese Substanz zu isolieren, wurde ihre Unlöslichkeit in Chloroform benützt und der ganze oben erhaltene krystallinische Rückstand durch mehrere Stunden mit Chloroform ausgekocht. Der in diesem Lösungsmittel unlösliche Teil ergab beim Umkrystallisieren aus Wasser monokline Prismen und Blättchen von gelber Farbe, die bei $195^{\circ}$ unter Zersetzung schmelzen.

Die Analyse der bei $120^{\circ}$ getrockneten Substanz ergab:

I. $0.2516 g$ Substanz gaben $0.5584 g \quad \mathrm{CO}_{2}$ und $0.1044 g \mathrm{H}_{2} \mathrm{O}$.

II. $0.2345 \mathrm{~g}$ Substanz gaben $0.5145 \mathrm{~g} \mathrm{CO}$ und $0.0936 \mathrm{~g} \mathrm{H}_{2} \mathrm{O}$.

In 100 Teilen:

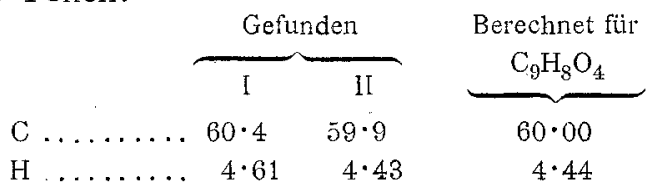

Die Resultate der Verbrennung stimmen gut mit der Formel der Kaffeesäure überein. Auch die schon früher angegebenen Eigenschaften der aus dem Zirbenharz erhaltenen Substanz sind mit denen der Kaffeesäure identisch. Der bei der Reinigung der Kaffeesäure erhaltene Chloroformextrakt gab beim Eindunsten eine geringe Menge einer dicken, braunen Masse, in der sich nach längerer Zeit kleine Krystalle ausschieden. Da letztere jedoch in zu geringer Quantität gewonnen wurden, konnte an eine Trennung und Isolierung nicht gedacht werden. Verschiedene Reaktionen weisen auf das Vorhandensein von Ferulasäure, so gibt Eisenchlorid einen braunen Niederschlag, Bleizucker eine zitronengelbe Fällung. ${ }^{2}$ Auch spricht der im Zirbenharz gefundene Methoxylgehalt für das Vorhandensein dieser Säure.

Die ursprünglich durch Abdestillieren des Alkohols erhaltene wässerige Lösung $A$ hatte einen an Vanillin erinnernden

1 Monatshefte für. Chemie, 12, 447, (1891).

2 Monatshefte für Chemie, 12, 452, (1891). 
Geruch, es wurde daher auch auf diesen Körper geprüft, indem die Lösung mit konzentrierter Salzsäure und Phloroglucinlösung versetzt wurde. Die auf diese Art erhaltene starke Rotfärbung weist auf das Vorhandensein von Vanillin. Bedeutend stärker trat diese Reaktion in dem Chloroformextrakt auf. Demnach dürfte in letzteren Vanillin und Ferulasäure vorhanden sein, da Vanillin in Chloroform leicht löslich ist. Die im Rohharz erhaltene Methoxylzahl dürfte also wohl zum größten Teil auf diese beiden Körper zurückzuführen sein. Von einer Isolierung des Vanillins wurde abgesehen, da die Menge eine zu geringe war und eine weitere Identifizierung nicht notwendig erschien. Vanillin wurde übrigens in verschiedenen Harzen bereits mehrfach nachgewiesen.

\section{Aufarbeitung von $B$.}

Nach Aufarbeitung der eingangs mit $A$ bezeichneten Lösung, wurde nun das geschmolzene Harz $B$ einer genaueren Untersuchung unterzogen.

Auch heute gelten noch die Worte G. Giamician's, ${ }^{1}$ daß man »bei Körpern von so komplizierter Natur, wie es die Harze sind, nur durch energisches Eingreifen Zersetzungsprodukte erhalten kann, welche chemisch gut charakterisiert sind . Auch hier wurde die Kalischmelze gemacht, da diese eben eine von den wenigen Methoden ist, welche es gestatten, zu gut charakterisierten Verbindungen $\mathrm{zu}$ kommen, die eventuell auch einen Einblick in die Konstitution des Harzkörpers gewähren. Ferner wurde das Harz auch einer schwächeren Verseifung unterworfen, um feststellen zu können, ob das Zirbenharz ein Resinol enthält.

\section{Einwirkung von schmelzendem Kali auf das ausgekochte Zirbenharz.}

$200 \mathrm{~g}$ ausgekochtes Harz wurden mit $1200 \mathrm{~g}$ Ätzkali in einer eisernen Schale zirka $1 \frac{1}{2}$ Stunden geschmolzen. Anfangs schmilzt das Harz in zähen Klumpen zusammen, welche auf dem geschmolzenen Ätzkali schwimmen, erst nach ungefähr

1 Berliner Berichte, 11, 269, (1878). 
einer halben Stunde fing es an, sich mit letzterem zu mischen. Bei andauerndem Rühren wurde nur schwaches Schäumen, wohl aber eine ziemlich starke Gasentwicklung beobachtet. Die hiebei auftretenden Gasblasen enthielten Wasserstoff. Als die starke Gasentwicklung nachgelassen hatte und nur mehr viele kleine Gasblasen zu sehen waren, war die Schmelze homogen. Gleichzeitig konnte ein Farbenumschlag rotbraun ins schwärzliche beobachtet werden. Als die Masse das deutliche Bestreben zum Einsinken zeigte, wurde die Schmelze unterbrochen.

Letztere wurde in Wasser gelöst und mit einem Überschuß von verdünnter Schwefelsäure versetzt. Hierbei schied sich eine beträchtliche Menge einer schwärzlichen Masse ab, von welcher die Flüssigkeit abfiltriert wurde. Die so erhaltene saure, von ausgeschiedenem Harz und dem größten Teil von Kaliumsulfat befreite Flüssigkeit wurde nun mit Äther ausgeschüttelt und dieser dann abdestilliert. Im. Kolben hinterblieb eine dicke, braune, ölige Masse, die stark nach Essigsäure und höheren Fettsäuren roch und nach zwei Tagen zu einer krystallinischen Masse erstarrte. Um die Trennung der Phenole von den Säuren vorzunehmen, wurde die erhaltene Krystallmasse in heißem Wasser gelöst, die Lösung mit kohlensaurem Baryt neutralisiert, letzterer abfiltriert und das Filtrat mit Äther ausgeschüttelt. Dieser Ätherauszug sei mit $A$ bezeichnet und die Aufarbeitung später beschrieben. Die auf die beschriebene Art von Phenolen befreite Flüssigkeit gab nach der Zersetzung mit Schwefelsäure an Äther die etwa vorhandenen Säuren ab (Ätherauszug $B$ ).

\section{Aufarbeitung von A (Phenole).}

Nach Abdestillieren des Äthers hinterblieb im Kolben eine dicke, braune, syrupartige Masse, die stark nach höheren Fettsäuren (wahrscheinlich Buttersäure) roch und nach einigen Tagen zu krystallisieren begann. Die Krystalle waren schön und groß ausgebildet und es konnten zwei verschiedene Krystallformen unterschieden werden. Die größeren derselben waren monoklinoedrische oft kombinierte Krystalle, die 
kleineren hingegen vierseitige Nadeln (Spieße) und Garben. Wie es sich später herausstellte, waren beide Krystallformen derselbe Körper. Diese Krystallmasse wurde nun abgesaugt. Die so erhaltenen Krystalle zeigten nach dem Umkrystallisieren aus Wasser den Schmelzpunkt $210^{\circ}$. Eisenchlorid gab in der wässerigen Lösung eine bräunliche Färbung, Bromwasser einen reichlichen Niederschlag. Alle diese Merkmale wiesen auf Paraoxybenzoesäure. ${ }^{1}$

Daß Paraoxybenzoesäure sowie der schon früher erwähnte Geruch nach Buttersäure hier im Extrakt, welcher die Phenole enthalten sollte, auftrat, ist weiter nicht verwunderlich, wenn man bedenkt, daß die Bariumsalze dieser Säuren zum Teil in Äther löslich sind. (Im Übrigen haben auch Hlasiwetz. und Barth ${ }^{2}$ diese Säuren zusammen mit Brenzkatechin gefunden.)

Die Mutterlauge, aus welcher die rohe Paraoxybenzoesäure krystallisierte, diente zur Gewinnung des Brenzkatechins. Erstere gab noch eine intensive Färbung mit Eisenchlorid, war dicker Konsistenz und daher nicht leicht krystallisierbar. Sie gab das Brenzkatechin nach nochmaligem Absättigen miẗ kohlensaurem Baryt (oder Natriumkarbonat) an Äther ab. Hierbei blieben die letzten Reste der anderen Verbindungen, wie noch etwa vorhandene Paraoxybenzoesäure und höhere Fettsäuren, welche noch in der Mutterlauge enthalten waren. ziemlich vollständig in der wässerigen Flüssigkeit zurück. Nach Abdestillieren des Äthers hinterblieb im Kolben eine braune Masse, die ein weit größeres Krystallisationsbestreben besaß als die frühere. Die entstandenen Krystalle waren rhombische Blättchen (Nadeln), die durch wiederholtes Umkrystallisieren aus Wasser gereinigt wurden. Das so erhaltene Produkt, das sich aber noch immer an der Luft ziemlich rasch bräunte, wurde durch Sublimation vollständig gereinigt. Der Körper besaß nun einen konstanten Schmelzpunkt von $104^{\circ}$ und gab in wässeriger Lösung folgende Reaktionen: Alkalien erzeugten sofort eine Braunfärbung, Eisenchlorid

\footnotetext{
1 Liebig's Annalen, 134, 271, (1865).

2 Liebig's Annalen, 134, 282, (1865).
} 
gab smaragdgrüne Färbung, die auf Zusatz von Sodalösung violett wurde.

Alle diese Eigenschaften wiesen auf Brenzkatechin.

Die Analyse der im Vakuumexsikkator gut getrockneten Substanz ergab:

I. $0.3218 \mathrm{~g}$ Substanz gaben $0.7704 \mathrm{~g} \mathrm{CO}_{2}$ und $0.1605 \mathrm{~g} \mathrm{H}_{2} \mathrm{O}$.

II. $0.1842 g$ Substanz gaben $0.4368 g \mathrm{CO}_{2}$ und $0.0905 \mathrm{~g} \mathrm{H}_{2} \mathrm{O}$.

In 100 Teilen:

\begin{tabular}{|c|c|c|c|}
\hline & \multicolumn{2}{|c|}{ Gefunden } & \multirow{2}{*}{$\begin{array}{l}\text { Berechnet für } \\
\mathrm{C}_{6} \mathrm{H}_{4}(\mathrm{OH})_{2}\end{array}$} \\
\hline & I & II & \\
\hline & $65 \cdot 29$ & $65 \cdot 31$ & $65 \cdot 5$ \\
\hline & $5 \cdot 5.4$ & $5 \cdot 51$ & $5 \cdot 46$ \\
\hline
\end{tabular}

\section{Aufarbeitung von B (Säuren).}

Nach Verdunsten des Äthers hinterblieb eine hellbraune, dickliche Masse, die stark nach Esșigsäure und höheren Fettsäuren roch. Die wässerige Lösung gab mit Eisenchlorid eine intensive grüne Färbung. Da Paraoxybenzoesäure schon früher bei der Gewinnung der Phenole aufgefunden worden war, so konnte hier ein Gemisch dieser Säure mit einer anderen, die mit Eisenchlorid eine grüne Färbung gibt, angenommen werden. Tatsächlich gab Bromwasser eine reichliche, schwach gelbe Fällung.

Zur Reinigung wurde die krystallinische Masse zuerst aus Alkohol und später aus Wasser umkrystallisiert. Man konnte wieder sehr gut zwei Krystallformen unterscheiden, es waren die schon früher beschriebenen Krystallkombinationen der Paraoxybenzoesäure $z u$ sehen und noch glänzende Nadeln einer zweiten Substanz. Durch wiederholtes Umkrystallisieren konnten die beiden Substanzen gut getrennt werden. Auch hier hatte die Paraoxybenzoesäure den Schmelzpunkt $210^{\circ}$ und gab mit Eisenchlorid eine braune Färbung.

$\mathrm{DaB}$ in beiden Fällen Paraoxybenzoesäure vorlag, ergab die Analyse der bei $120^{\circ}$ getrockneten Verbindung.

I. $0.1495 g$ Substanz gaben $0.3315 g \mathrm{CO}_{2}$ und $0.0688 \mathrm{~g} \mathrm{H}_{2} \mathrm{O}$.

II. $0 \cdot 1434 g$ Substanz gaben $0 \cdot 3183 g \mathrm{CO}_{2}$ und $0 \cdot 0568 g \mathrm{H}_{2} \mathrm{O}$. 
In 100 Teilen:

$$
\begin{aligned}
& \text { Gefunden Berechnet für } \\
& \text { I. } \quad \text { II. } \quad \underbrace{\mathrm{C}_{7} \mathrm{H}_{6} \mathrm{O}_{3}}_{7} \\
& \begin{array}{llll}
\text { C } \ldots \ldots .66 & 60.47 & 60 \cdot 74 & 60 \cdot 86
\end{array} \\
& \text { H ..... } 5 \cdot 1 \quad 4 \cdot 4 \quad 4 \cdot 34
\end{aligned}
$$

Der zweite Körper gab mit Eisenchlorid eine intensive blaugrüne Färbung, welche auf Zusatz von etwas Soda dunkelrot wurde. Der Schmelzpunkt war konstant bei $199^{\circ}$.

Die Analyse der bei $120^{\circ}$ getrockneten Verbindung: ergab:

$0 \cdot 1564 g$ Substanz gaben $0.3116 g \mathrm{CO}_{2}$ und $0.0559 g \mathrm{H}_{2} \mathrm{O}$.

In 100 Teilen:

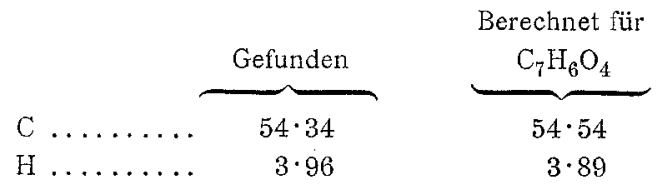

Die Reaktionen wie auch die Resultate der Verbrennung stimmen gut für Protokatechusäure.

Die schon früher erwähnte Fällung mit Bromwasser aus dem Gemenge der beiden oben untersuchten Säuren wurde abgesaugt und einige Male aus verdünntem Alkohol umkrystallisiert und eine weiße verfilzte Masse erhalten, die alle Eigenschaften des Tribromphenols besa $\beta$ und bei $91^{\circ}$ schmolz. Da dieser Körper aus der früher gefundenen Paraoxybenzoesäure entsteht und seine Eigenschaften mit denen des Tribromphenols identisch waren, wurde von einer weiteren Analyse dieser Substanz abgesehen.

Auch das Filtrat des abgesaugten Bromierungsproduktes. konnte einer Nachprüfung dadurch unterzogen werden, daß. es mit Natriumamalgam entbromt, hierauf mit Schwefelsäure angesäuert und mit Äther ausgeschüttelt wurde. Nach dem Abdestillieren des letzteren konnte wieder die Substanz erhalten werden, welche die charakteristischen Eigenschaften der Protokatechusäure besaß. Mithin war mit Sicherheit nachgewiesen, daß das Säuregemenge tatsächlich aus Paraoxybenzoesäure und Protokatechusäure bestand. 
Was noch den schon öfters erwähnten Geruch nach höheren flüchtigen Fettsäuren betrifft, so kann festgestellt werden, daß ein starker Geruch von Buttersäure ${ }^{1}$ vorhanden war. Eine Isolierung dieser Fettsäuren durch Destillation der wässerigen Lösung war nicht erzielbar, da die vorliegenden Mengen $z u$ einer genauen Untersuchung nicht ausreichten.

\section{Trennung des Zirbenharzes in $\alpha$ - und $\beta$-Harz.}

Auch das Zirbenharz läßt sich ähnlich wie die Überwallungsharze der Schwarzföhre, Lärche und Fichte durch Äther in $\alpha$ - und $\beta$-Harz trennen, doch ist hier das $\beta$-Harz nur mit wenigen Prozenten vertreten. $200 \mathrm{~g}$ ausgekochtes Harz - eingangs der Arbeit mit $B$ bezeichnet - wurde mit Äther übergossen und einige Tage stehen gelassen. Nach dieser Zeit wurde abfiltriert, der Äther entfernt und das hinterbliebene o-Harz durch Alkohol aufgenommen und in mit Salzsäure angesäuertes Wasser gegossen. Im Wasser schied sich das Harz als eine lichte, schmierige Masse $a b$, die nach längerem Stehen und öfterem Durchwaschen mit kaltem Wasser etwas konsistenter gemacht werden konnte, Um das Harz ganz frei von Alkohol zu erhalten, wurde es noch wiederholt mit Wasser ausgekocht und nach dieser Operation schied es sich als eine lichtbraune Masse ab, die beim Ausziehen in Fäden seidenartig glänzte. Das $\alpha$-Harz verlor auch nach längerer Zeit seine zähe Konsistenz nicht, sondern blieb eine teigartige Masse.

Der in Äther ungelöst gebliebene Teil, also das $\beta$-Harz, wurde zur Reinigung in Alkohol aufgenommen und in mit Salzsäure angesäuertes Wasser eingetragen. Das in Flocken ausgeschiedene Harz wurde abfiltriert und getrocknet. Anfangs war das im Wasser ausgeschiedene $\beta$-Harz schwach rosa gefärbt, nach dem Trocknen stellte es ein rotbraunes Pulver dar, welches alle Reaktionen zeigte, die Tschirch für Tannole angibt; so erhält man beim Versetzen seiner alkoholischen

1 Hlasiwetz und Barth haben ebenfalls einen derartigen Geruch bei der Aufarbeitung der Kalischmelze von Benzoeharz konstatieren können (siehe Liebigs Annalen, 134, 278, (1865). 
Lösung mit Eisenchlorid einen braunschwarzen, mit Kaliumbichromat einen rotbraunen und mit Bleizucker einen hellbraunen Niederschlag.

Von den angewandten $200 \mathrm{~g}$ Harz konnten nur $4 g$ - - Harz gewonnen werden, während alles andere $\alpha$-Harz war. Dies würde daher einem Verhältnis von zirka $98 \%$ a- und $2 \%$ $\beta$-Harz entsprechen.

Die einzelnen Harzkomponenten wurden auch noch auf ihren Metoxylgehalt geprüft.

Das ausgekochte, noch unzerlegte Grundharz zeigte einen Methylgehalt von:

$$
7 \cdot 12 \text { Gewichtsteilen } \mathrm{CH}_{3} \text { in } 1000 \text { Teilen, }
$$
das $\alpha$-Harz gab $\ldots 0 \cdot 84$ Gewichtsteile $\mathrm{CH}_{3}$ in 1000 Teilen, und das $\beta$-Harz: $.17 \cdot 4$ Gewichtsteile $\mathrm{CH}_{3}$ in 1000 Teilen.

Daß der $\beta$-Harz-Gehalt ein so auffallend geringer war, dürfte vielleicht darin seinen Grund haben, daß das verwendete Zirbenharz zum weitaus größten Teil aus einem über die ganze Borke verteilten zähflüssigen, weichen Balsam bestand, der wahrscheinlich noch nicht lange der oxydierenden Wirkung des Luftsauerstoffes ausgesetzt war.

\section{Versuch zur Gewinnung eines Resinols.}

Pino- und Lariciresinol konnten von Bamberger und Landsied $1^{1}$ aus den Überwallungsharzen der Fichte, Schwarzföhre, und Lärche gewonnen werden. Um ein Resinol aus dem Zirbenharz zu erhalten, wurde das ausgekochte Harz in Alkohol gelöst, diese Lösung auf dem Wasserbade erwärmt und nun soviel Ätzkali hinzugefügt, bis die Solution $z u$ einem einheitlichen Kuchen erstarrte, der dann von der vorhandenen Mutterlauge abgenutscht wurde. Diese Abscheidung war nicht wie beim Pino- und Lariciresinolkalium krystallinisch, sondern vollständig amorph und harzartig. Die erhaltene Kaliumverbindung ist in Alkohol im Gegensatz zu den Kaliumverbindungen des Pino- und Lariciresinols leicht und vollständig löslich und wird durch Eingießen in mit Salzsäure angesäuertes Wasser

1 Monatsliefte für Chemie, 15, 507, (1894). 
zerlegt. Es schied sich als ein brauner, harziger Körper ab, der trotz aller Bemühungen nicht zum Krystallisieren gebracht. werden konnte.

Obwohl nun die Zirbenkiefer in dieselbe Pflanzenfamilie gehört wie die Schwarzkiefer, konnte aus dem Harz derselben kein Resinol gewonnen werden.

Nach obiger Untersuchung des Zirbenharzes konnten folgende Substanzen festgestellt werden:

I. Im präformierien Zustande waren enthalten:

1. Kaffeesäure $\mathrm{C}_{6} \mathrm{H}_{3}(\mathrm{OH})_{2} \mathrm{CH}=\mathrm{CH}-\mathrm{COOH}$.

2. Ferulasäure $\mathrm{C}_{6} \mathrm{H}_{3}(\mathrm{OH})\left(\mathrm{OCH}_{3}\right) \mathrm{CH}=\mathrm{CH}-\mathrm{COOH}$.

3. Vanillin $\mathrm{C}_{6} \mathrm{H}_{3}(\mathrm{OH})\left(\mathrm{OCH}_{3}\right)-\mathrm{CHO}$.

II. In der Kalischmelze wurde gefunden:

1. Essigsäure, höhere flüchtige Fettsäuren (Buttersäure).

2. Paraoxybenzosäure $\mathrm{C}_{6} \mathrm{H}_{4}(\mathrm{OH}) \mathrm{COOH}$.

3. Protokatechusäure $\mathrm{C}_{6} \mathrm{H}_{3}(\mathrm{OH})_{2} \mathrm{COOH}$.

4. Brenzkatechin $\mathrm{C}_{6} \mathrm{H}_{4}(\mathrm{OH})_{2}$.

Es ist uns eine angenehme Pflicht, Herrn Prof. Dr. F. Krasser für seinen freundlichen Beitrag bestens zu danken. Dem Herrn Vorstand der k. k. Forst- und Domänendirektion für Tirol und Vorarlberg, Hofrat Friedrich Freiherrn D a ublebsky v. Sterneck, Herrn Forst- und Domänenverwalter Josef Lüftenegger in Prutz sowie Herrn Prof. Dr. Karl M eusburger in Brixen sagen wir für die Beschaffung des so schwer erhältlichen Überwallungsharzes der Zirbe den verbindlichsten Dank.

II. Zur Kenntnis des Lariciresinols.

\section{Zinkstaubdestillation von Lariciresino1.}

Zur weiteren Ermittlung der Konstitution des aus dem Harz der Lärche gewonnenen Lariciresinols wurde dieses auch der Zinkstaubdestillation unterworfen, um der Ermittlung: des Grundkohlenwasserstoffes näher zu treten. 
Lariciresinol wurde in Portionen von je $15 \mathrm{~g}$ mit der zehnfachen Menge Zinkstaub verrieben und in einem weiten Verbrennungsrohre im Wasserstoffstrom destilliert. Die gasförmigen Destillationsprodukte wurden zuerst in einer durch fließendes Wasser gekühlten Vorlage kondensiert und die schwerer zu verdichtenden Produkte noch in eine durch feste Kohlensäure gekühlte Vorlage geleitet. Ein geringer Teil der flüchtigsten Produkte konnte auf diesem Weg nicht zur Kondensation gebracht werden und ging verloren. Auf diese Art wurde die ganze zur Verfügung stehende Menge von $150 \mathrm{~g}$ Lariciresinol der Destillation unterworfen.

Beim Erhitzen entwichen anfangs weiße Dämpfe und später kamen ölige Tropfen nach. Aus der oben angegebenen Menge Lariciresinol wurde ein Destillat von zirka $40 \mathrm{~cm}^{3}$ erhalten. Dasselbe war dunkelbraun, stark riechend und schwerer als Wasser. Zur ersten Trennung der darin enthaltenen Substanzen wurde es mit Wasserdampf destilliert und diese Operation solange fortgesetzt, bis keine Öltropfen mehr übergingen. Es wurde so ein gelb gefärbtes Öl - $A$-, welches leichter als Wasser war, erhalten und es blieb eine schwarzbraune, teerartige, dicke Masse zurück, welche, nachdem sie von Wasser befreit war, einen beträchtlichen Tei] an Äther abgab. Von dieser Lösung blieb nach Abdestillieren des letzteren eine braune, schwere, ölartige Flüssigkeit - $B-$ zurück, deren Geruch an Naphthalin und höhere Phenole erinnerte. Der mit Äther ausgezogene schwarze, pechartige Destillationsrückstand wurde wegen seiner geringen Menge nicht weiter berücksichtigt.

I. Die mit Wasserdampf flüchtigen Produkte - A -- hatten einen starken, an höhere Phenole erinnernden Geruch, wurden mit Natronlauge gereinigt und hierauf mit Äther ausgeschüttelt. Nach Abdestillieren des letzteren hinterblieb eine gelbe, nur noch schwach brenzlich riechende Flüssigkeit, die mit dem Teil $B$, nachdem dieser ebenfalls mit Natronlauge behandelt worden war, vereinigt und gemeinsam einer fraktionierten Destillation unterworfen wurde. Jede der erhaltenen Fraktionen wurde nochmals destilliert, um die einzelnen Substanzen zwecks Identifizierung möglichst rein zu erhalten. 
Es konnten so nachfolgende Produkte der Zinkstaubdestillation festgestellt werden:

1. Die am niedersten siedende. Fraktion zeigte bei der Rektifikation den Siedepunkt $111^{\circ}$. Zur weiteren Feststellung: wurde ein Nitroprodukt durch Eintragen der Fraktion in rauchende Salpetersäure hergestelit. Es wurden gelbe, monokline Nadeln erhalten, die einen Schmelzpunkt von zirka $70^{\circ}$ hatten ${ }^{1}$. Somit war diese Fraktion als Toluol zu erkennen.

2. Der Kohlenwasserstoff der bei zirka $140^{\circ}$ übergehenden Fraktion konnte als Xylol erkannt werden, doch war die Menge eine $\mathrm{zu}$ geringe, um noch $\mathrm{zu}$ bestimmen, welches Isomere vorlag, auch war das erhaltene Destillat nicht frei von Toluol.

3. Diese Fraktion hatte einen Siedepunkt von zirka $152^{\circ}$. Wegen Mangel an einer genügenden Menge derselben konnte eine Identifizierung nicht mit Erfolg durchgeführt werden, jedoch scheint Cumol oder Metaäthylmethylbenzol vorzuliegen.

4. Aus dem zwischen 200 bis $220^{\circ}$ übergehenden Destillat schieden sich nach einigen Tagen weiße Krystalle aus. Durch fraktioniertes Ausfrieren mit Hilfe von Kohlensäureschnee und darauffolgendes rasches Abschleudern durch eine feine Siebplatte gelang die Isolierung des ausgeschiedenen Körpers $(a)$ recht befriedigend. Das Filtrat (b) war ein gelbliches, schweres Öl.

a. Um die früher erwähnte stark naphthalinartig riechende Substanz von den letzten Spuren anhaftenden Öls zu befreien, wurde sie abgepreßt, mit etwas Alkohol gewaschen und die so gereinigte Verbindung in möglichst wenig siedendem Alkohol gelöst. Beim Abkühlen fiel der Körper in schönen weißen Krystallen (Blättchen) aus, dessen Schmelzpunkt $79^{\circ}$ betrug. Eine ätherische Pikrinsäurelösung fälite aus der ätherischen Lösung des Körpers ein gelbes Pikrat, das bei $148^{\circ}$ schmolz. Die Substanz gab folgende Reaktionen:

1. Mit Wasserstoffsuperoxyd: Intensiv grün.

1 Trägt man Toluol in rauchende Salpetersäure ein, so entsteht hauptsächlich 2, 4-Dinitrotoluol. 
2. Mit Benzaldehyd und Schwefelsäure ene starke Rotfärbung, die besonders beim Erwärmen auf dem Wasserbade rasch auftrat.

3. Mit Benzaldehyd und Schwefelsäure: Fuchsinrot.

Alle angegebenen Eigenschaften und Reaktionen stimmen mit Naphthalin sehr gut überein.

b. Aus dem früher erhaltenen, gelben, öligen Filtrat wurde eine Fraktion erhalten, die nach wiederholtem Destillieren bei $232^{\circ}$ überging. Sie hatte einen naphthalinartigen Geruch und wurde nach den Angaben von G. Ciamician ${ }^{1}$ geprüft, da Methylnaphthalin vermutet wurde.

Das mit Alkohol aufgenommene Destillat wurde mit einer gesättigten, warmen Lösung von Pikrinsäure versetzt. Beim Erkalten schieden sich feine, verfilzte, orangegelbe Nadeln aus. Das erhaltene Pikrat wurde mehreremale aus Alkohol umkrystallisiert und zeigte sehr genau den Schmelzpunkt von $116^{\circ}$, der mit dem von G. Ciamician angegebenen 116 bis $117^{\circ}$ übereinstimmt. Aus dieser Pikrinsäureverbindung konnte durch Erwärmen der Lösung mit Ammoniak das Methylnaphthalin wieder frei gemacht werden, welches sich in Form öliger Tropfen abschied.

5. Die letzte, zwischen 260 bis $290^{\circ}$ übergehende Fraktion konnte wegen der zu geringen Menge nicht identifiziert werden, doch dürfte ein höheres Homologes von Naphthalin vorliegen.

II. Die eingangs (unter I) zum Reinigen der Kohlenwasserstoffe verivendete Natroniauge wurde angesäuert und mit Äther ausgeschüttelt. Nach Abdestillieren des letzteren hinterblieb eine geringe Menge braunen, kreosotartigen Öls. Der Geruch nach Guajakol war unverkennbar, doch war leider die Menge zu gering, um dasselbe zu isolieren.

Von den Produkten, die somit bei der pyrogenen Reduktion des Lariciresinols mit Zinkstaub gebildet worden waren, sind nachgewiesen worden:

Toluol, Xylol, Cumol (?), Phenole (Guajakol), Naphthalin und Methylnaphthalin.

1 Berliner Berichte, 11, 272, (1878). 


\section{Einwirkung von Ozon auf Lariciresinol.}

Ein Versuch, das Verhalten von Lariciresinol gegen Ozon zu prüfen, führte nicht zur Isolierung von Spaltungsprodukten, doch konnte immerhin qualitativ die Bildung eines Ozonides nachgewiesen werden. Es wurde nach den Angaben von Harries gearbeitet und die von ihm empfohlene Apparatur verwendet. ${ }^{1}$ Als Lösungsmittel diente wasserfreies Chloroform. Stets trat beim Ozonisieren eine starke, gelbe bis gelbbraune Färbung der ursprünglich farblosen Lösung ein und an den Wänden des Gefäßes schied sich ein harzartiger gelbbrauner Belag ab, der auch aus verschiedenen Lösungsmitteln nicht krystallinisch erhalten werden konnte. Beim Durchschütteln der Reaktionsflüssigkeit mit Wasser wurde dieses beim Versetzen mit Titansulfat intensiv gelb gefärbt. Somit war ein Ozonid vorhanden, das unter Bildung von Wasserstoffsuperoxyd gespalten wird.

\section{Zur Kenntnis des Pinoresinols.}

\section{Einwirkung alkoholischer Salzsäure auf Pinoresinol.}

Nach früheren Arbeiten² kommt dem aus dem Harz der Fichte und Schwarzföhre gewonnenen Pinoresinol die empirische Formel $\mathrm{C}_{19} \mathrm{H}_{20} \mathrm{O}_{6}$ zu. Auch ist in diesen Publikationen bereits nachgewiesen worden, daß das Pinoresinol zwei freie Hydroxylgruppen enthält, da es ein Diacethyl, ein Dibenzoylund ein Dimethylprodukt liefert. Ebenso ist festgestellt, daß diese Verbindung zwei Methoxylgruppen enthält.

Es war nun von Interesse $z u$ untersuchen, ob Pinoresinol $\mathrm{C}_{16} \mathrm{H}_{10} \mathrm{O}_{2}(\mathrm{OH})_{2}\left(\mathrm{OCH}_{3}\right)_{2}$ so wie Lariciresinol ein Anhydroprodukt liefert. Würde Pinoresinol in gleicher Weise ein solches Derivat geben, so wäre zu erwarten gewesen, daß Anhydropinoresinol in Kalilauge nicht unzersetzt löslich wäre und weder ein neues Diacethyl-, noch ein Dimethylprodukt geben duifte, da beim Austritt von $1 \mathrm{Mol}$ Wasser aus den zwei freien

1 Liebig's Annalen, 343, 339, (1905).

2 H. Hermann, Monatshefte für Chemie, 23, 1022, (1902). 
Hydroxylgruppen keine derartigen Substanzen mehr zu erwarten waren, oder daß hiebei wieder Pinoresinol regeneriert wird.

Zur Herstellung eines Anhydroproduktes wurde nach dem E. Fischer'schen Esterifizierungsverfahren ${ }^{1}$ mit äthylalkoholischer Salzsäure gearbeitet. $3 g$ Pinoresinol wurden mit $24 \mathrm{~cm}^{3}$ Alkohol gelöst, $2 \mathrm{~cm}^{3}$ konzentrierter Salzsäure zugefügt und dann durch 6 Stunden auf dem Wasserbade unter dem Rückflußkühler erhitzt. Nach einiger Zeit färbt sich die anfangs farblose Lösung dunkelbraun. Nach 24 Stunden hatte sich ein rotbrauner Körper ausgeschieden, der abfiltriert und mit wenig Alkohol nachgewaschen wurde. Diese Operation wurde einige Male mit verschiedenen Konzentrationen und verschieden langer Kochdauer wiederholt. Hiebei wurde jedoch immer derselbe Körper erhalten. Dieser war dunkelbraun, amorph und konnte trotz wiederholter Versuche nicht krystallisiert erhaiten werden. Ein Schmelzpunkt wurde nicht beobachtet. Die rotbraune Farbe ist dem Körper eigen und konnte derselbe auch durch wiederholtes Behandeln der Lösung mit Knochenkohle nicht heller erhalten werden. Die Methoxylbestimmung gab folgende Werte:

In 1000 Teilen:

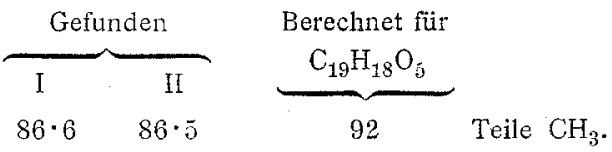

Die rote amorphe Substanz wurde bei $105^{\circ}$ getrocknet. Die Analyse gab folgende Werte:

I. $0.2064 \mathrm{~g}$ Substanz gaben $0.5262 \mathrm{~g} \mathrm{CO}_{2}$ und $0.1154 \mathrm{~g} \mathrm{H}_{2} \mathrm{O}$.

II. $0.2341 \mathrm{~g}$ Substanz gaben $0.5945 \mathrm{~g} \mathrm{CO}$ und $0.1213 \mathrm{~g} \cdot \mathrm{H}_{2} \mathrm{O}$.

III. $0 \cdot 2542 g$ Substanz gaben $0.6499 g \mathrm{CO}_{2}$ and $0.1305 g \mathrm{H}_{2} \mathrm{O}$.

In 100 Teilen:

\begin{tabular}{|c|c|c|c|c|}
\hline \multirow[t]{2}{*}{ 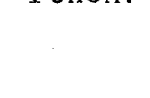 } & \multicolumn{3}{|c|}{ Gefunden } & \multirow{2}{*}{$\begin{array}{c}\text { Berechnet für } \\
\mathrm{C}_{19} \mathrm{H}_{18} \mathrm{O}_{\mathfrak{5}}\end{array}$} \\
\hline & I & II & III & \\
\hline $\mathrm{C}$ & $69 \cdot 53$ & $69 \cdot 2$ & $69 \cdot 73$ & $69 \cdot 94$ \\
\hline$H \ldots \ldots$ & $6 \cdot 26$ & $5 \cdot 7$ & $5 \cdot 70$ & $5 \cdot 52$ \\
\hline
\end{tabular}

1 B., (1895), p. 3252 . 
Aus diesen Analysenresultaten läßt sich die Formel $\mathrm{C}_{19} \mathrm{H}_{18} \mathrm{O}_{5}$ berechnen. Es ist also 1 Mol Wasser ausgetreten und der vorliegende neu erhaltene Körper könnte vielleicht als ein Anhydroprodukt des Pinoresinols betrachtet werden.

Dieses Anhydroprodukt dürfte schon in einer früheren Arbeit ${ }^{1}$ beobachtet worden sein, da Pinoresinol in konzentrierter Schwefelsäure mit intensiv roter Farbe löslich ist und sich nach einiger Zeit als rotbrauner Körper ausscheidet. Konzentrierte Salzsäure verhält sich ähnlich. In dieser löst sich nämlich Pinoresinol sehr leicht auf; nach kurzer Zeit scheidet sich jedoch ein weißer Körper aus, der beim Erwärmen rotbraun wird.

Das erhaltene Anhydroprodukt löst sich gegen Erwarten in Kalilauge. Die Methylierung desselben durch Behandeln mit Dimethylsulfat in alkalischer Lösung gelang nicht. Hingegen liefert das Anhydroprodukt beim Acetylieren mit Acetylchlorid ein Acetylprodukt. Das Anhydroprodukt löst sich leicht, besonders beim Erwärmen auf dem Wasserbade in Acetylchlorid auf. Beim Eingießen dieser Lösung in Wasser scheidet. sich ein Öl ab, das bald krystallinisch erstarrte. Die erhaltene Krystallmasse wurde abfiltriert und aus Alkohol umkrystallisiert und stellte einen schwachgelben, in feinen Nadeln. krystallisierenden Körper dar.

Die Analyse der bei $100^{\circ}$ getrockneten Substanz ergab:

I. $0.1299 \mathrm{~g}$ Substanz gaben $0.3049 \mathrm{~g} \mathrm{CO}_{2}$ und $0.0690 \mathrm{~g} \mathrm{H}_{2} \mathrm{O}$.

II. $0.2245 \mathrm{~g}$ Substanz gaben $0.5305 \mathrm{~g} . \mathrm{CO}_{2}$ und $0.1182 \mathrm{~g} \mathrm{H}_{2} \mathrm{O}$.

In 100 Teilen:

\begin{tabular}{|c|c|c|c|}
\hline & \multicolumn{2}{|c|}{ Gefunden } & \multirow{2}{*}{$\begin{array}{c}\text { Berechnet für } \\
\mathrm{C}_{19} \mathrm{H}_{18} \mathrm{O}_{6}\left(\mathrm{C}_{2} \mathrm{H}_{3} \mathrm{O}\right)\end{array}$} \\
\hline & I & II & \\
\hline & $64 \cdot 02$ & $64 \cdot 44$ & $64 \cdot 48$ \\
\hline & $.5 \cdot 9$ & $5 \cdot 85$ & $5 \cdot 6$ \\
\hline
\end{tabular}

Zur weiteren Identifizierung wurde noch eine Acetylbestimmung nach Wenzel ${ }^{2}$ ausgeführt. $0.229 g$ Substanz. gab ein essigsaures Destillat, das $12 \mathrm{~cm}^{3}$ zehntelnormale Kalilauge verbrauchte.

1 Nonatshefte für Chemie, 15, 508, (1894).

2. Monatshefte für Chemie, 18, 659, (1897). 
In 100 Teilen:

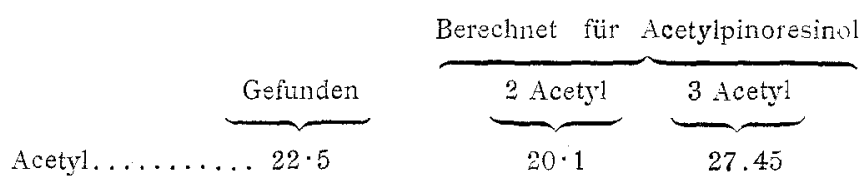

Die Verbrennung wie die Acetylbestimmung ergab also, daß aus dem Anhydropinoresinol wieder das bekannte Diacetylpinoresinol erhalten wurde.

Bei der anfangs angegebenen Operation der Herstellung des Anhydroproduktes durch Erhitzen mit alkoholischer Salzsäure trat stets ein deutlicher kreosotähnlicher Geruch auf. Es wurde daher versucht, eine Spaltung des Pinoresinols aut diesem Wege zu erreichen. Da auf diese Weise keine genügende Menge des riechenden Stoffes erhalten werden konnte, wurde mit stärkerer Salzsäure und verschieden langer Kochdauer gearbeitet. Auch ein Versuch im Bombenrohr führte nicht zu einer größeren Menge dieser stark riechenden Substanz. Es scheint bei diesen Versuchen nur zur Spaltung eines ganz geringen Teiles des Ausgangsmateriales zu kommen. Es wurde weiters versucht, diesen kreosotartig riechenden Körper durch Destillation des Reaktionsproduktes im Wasserdampfstrom zu isolieren. Aus dem Destillat konnte eine ganz geringe Menge durch Ausschütteln mit Äther erhalten werden. Die erhaltene Menge wurde mit etwas Alkohol aufgenommen und gab mit Eisenchlorid eine grüne Färbung. Das Produkt besaß einen dem Guajakol äußerst ähnlichen Geruch. 